\title{
FREGEAN SUBTRACTIVE VARIETIES WITH DEFINABLE CONGRUENCE
}

\author{
PAOLO AGLIANO
}

(Received 24 February 1999; revised 21 September 2000)

Communicated by B. A. Davey

\begin{abstract}
In this paper we investigate subtractive varieties of algebras that are Fregean in order to get structure theorems about them. For instance it turns out that a subtractive variety is Fregean and has equationally definable principal congruences if and only if it is termwise equivalent to a variety of Hilbert algebras with compatible operations. Several examples are provided to illustrate the theory.
\end{abstract}

2000 Mathematics subject classification: primary 08A99, $08 \mathrm{~A} 30$.

Keywords and phrases: Fregean, subtractive variety, definable congruences.

\section{Introduction}

$A$ few words on general notation. If $\mathbf{A}$ is an algebra, then $1_{A}$ and $0_{A}$ always denote the largest and the smallest congruence on $\mathbf{A}$. If $X \subseteq A^{2}$, then $\vartheta_{A}(X)$ is the smallest congruence containing all pairs in $X$. If $X=\{a, b\}$, we write $\vartheta_{A}(a, b)$. Finally, if $Y \subseteq A, \operatorname{Sub}_{\mathbf{A}}(Y)$ is the smallest subalgebra of $\mathbf{A}$ containing $Y$.

The idea of distinguishing between Fregean and non-Fregean logics is mainly due to Roman Suszko and it originates with a proposal for formalizing the logical system of Wittgenstein's Tractatus. The main feature of a non-Fregean logic is the distinction made between denotation of a sentence and its truth value. This distinction, in Suszko's work, was embodied by a new binary connective called identity. Connecting two sentences via the identity connective expresses the fact that they refer to the same thing (that is, they are 'identical'), while the ordinary equivalence connective expresses merely the fact that they have the same truth value. A Fregean logic is simply a logic in which identity and equivalence coincide.

(C) 2001 Australian Mathematical Society 0263-6115/2001 \$A2.00+0.00 
We can give this property a more transparent algebraic meaning. Consider a classical logic $L$ and let $B$ be the Lindenbaum algebra of $L$. Then $B$ is a Boolean algebra and, via the strong completeness theorem, a classical logic can be interpreted fully in the variety of Boolean algebras (that is, the variety of Boolean algebras is the equivalent algebraic semantics [8] of classical logic). If $p, q \in \mathscr{B}$, then $p$ and $q$ are identical in the above sense, if $p=q$ in B. On the other hand, if $p$ and $q$ have the same truth value, then $p$ and $q$ generate the same Boolean filter in $\mathscr{B}$. But for any Boolean algebra $\mathbf{A}$ and $a, b, \in A$

$$
\vartheta_{\mathrm{A}}(1, a)=\vartheta_{\mathrm{A}}(1, b) \text { if and only if } a=b .
$$

This, via the usual description of Boolean filters and congruence regularity at 1 ,implies that two elements generate the same Boolean filter if and only if they are equal. Hence a classical logic is Fregean, a well-known fact.

The normal modal logic $\$ 4$ is an example of a non-Fregean logic. The Lindenbaum algebra I of S4 is an interior algebra and the variety of interior algebras is the equivalent algebraic semantics for S4. For $p, q \in I$, being identical again means $p=q$ and having the same truth value means that $p$ and $q$ generate the same open filter in $\mathbf{I}$. But for any interior algebra $\mathbf{A}$ and $a, b \in A$

$$
\vartheta_{\mathrm{A}}(1, a)=\vartheta_{\mathrm{A}}(1, b) \text { if and only if } a^{\circ}=b^{\circ},
$$

where ${ }^{\circ}$ denotes the interior operation. Since, in general, $a^{\circ} \neq a$, via the description of open filters and congruence regularity at 1 , one sees that $\$ 4$ is non-Fregean (for the axiomatization and the main properties of interior algebras the reader can consult [7] or [6]).

Since in many cases being Fregean (or non-Fregean) is a purely algebraic matter, one may wish to abstract from the logical origin of the notion. To be more precise let $\mathscr{V}$ be a pointed variety (and this time 0 be the distinguished element) and let $\mathbf{A} \in \mathscr{V}$. Then one is led naturally to the study of the equivalence relation $\approx_{\mathrm{A}}$ on $A$ defined by

$$
a \approx_{\mathrm{A}} b \text { if and only if } \vartheta_{\mathrm{A}}(0, a)=\vartheta_{\mathrm{A}}(0, b) .
$$

The pointed varieties for which $\approx_{A}=0_{A}$ for any algebra $\mathbf{A}$ have been introduced by Büchi and Owens in [11] and have attracted some attention recently $[12,18,20]$. However, there is an ongoing debate among the specialists, regarding what these varieties should be called. In particular the question is: Do they deserve to be called Fregean? The name seems entirely plausible. However, in [18] it was observed that in many cases the assumption of point-regularity is crucial in proving that a variety is 'Fregean'. On the other hand, some maintain (with some reason) that from the point of view of connections with logic it is more natural not to assume point regularity. We do not wish to take sides here, but nonetheless a choice must be made. Since this 
investigation was mainly inspired by [18] we have decided to adopt their terminology. Thus we say that a pointed variety $\mathscr{V}$ is congruence orderable if $\approx_{\mathrm{A}}=0_{\mathrm{A}}$ for each $\mathrm{A} \in \mathscr{V}$. A pointed variety is Fregean if it is congruence orderable and point regular. Fregean varieties are known to admit a relevant structure theorem in two cases.

- If a variety $\mathscr{V}$ is Fregean and the join of any two principal ideals is principal, then it is termwise equivalent to a variety of Skolem semilattices with compatible operations [20]. This means that there are terms $m(x, y)$ and $e(x, y)$ of $\mathscr{V}$ such that for any algebra $\mathbf{A} \in \mathscr{V},\langle A, m, e, 0\rangle$ is a Skolem semilattice and $\operatorname{Con}(\mathbf{A})=$ $\operatorname{Con}(\langle A, m, e\rangle)$. Therefore any such variety has equationally definable principal congruences. In [18] it is shown that this class coincides with the class of arithmetical (that is, congruence permutable and congruence distributive) congruence orderable varieties. Boolean algebras, Heyting algebras and Brouwerian semilattices are the main examples of varieties of this kind.

- If a variety $\mathscr{V}$ is congruence orderable and congruence permutable, then it is Fregean and there is a term $e(x, y)$ of $\mathscr{V}$ (said to be an equivalential term) such that for any $\mathbf{A} \in \mathscr{V},\langle A, e, 0\rangle$ is an equivalential algebra [18]. Moreover any basic operation $f\left(x_{1}, \ldots, x_{n}\right)$ of A satisfies a weak form of compatibility

$$
e\left(f\left(a_{1}, \ldots, a_{n}\right), f\left(b_{1}, \ldots, b_{n}\right)\right) \in \bigvee_{i=1}^{n}\left(e\left(a_{i}, b_{i}\right)\right)_{\mathbf{A}}
$$

The variety of Boolean groups is an example of a variety of this kind that is not congruence distributive (and hence does not have equationally definable principal congruences).

There is an important example of a Fregean variety that falls outside either one of the above classes. The variety of Hilbert algebras is Fregean, congruence distributive, has equationally definable principal congruences but it is not congruence permutable. We deal with it in Section 3.

\section{Preliminaries}

2.1. Ideal theory All classes of algebras we are going to consider are pointed, that is, there is a constant in their type set which is always denoted by 0 . If $\mathscr{K}$ is a class of similar algebras, a term $p\left(x_{1}, \ldots, x_{m}, y_{1}, \ldots, y_{n}\right)$ is a $\mathscr{K}$-ideal term in $\vec{y}$ (and we write $p(\vec{x}, \vec{y}) \in \mathbf{I T}_{\mathscr{K}}(\vec{y})$ ) if the identity $\mathrm{p}(\vec{x}, 0, \ldots, 0) \approx 0$ holds in $\mathscr{K}$. A nonempty subset $I$ of $\mathbf{A} \in \mathscr{K}$ is a $\mathscr{K}$-ideal of $\mathbf{A}$ if for any $p(\vec{x}, \vec{y}) \in \mathbf{T} \mathbf{T}_{\mathcal{X}}(\vec{y})$, for $\vec{a} \in A$ and $\vec{b} \in I, p(\vec{a}, \vec{b}) \in I$. Under inclusion, the set $\mathrm{I}_{\mathscr{X}}(\mathbf{A})$ of all $\mathscr{K}$-ideals of $\mathbf{A}$ is an algebraic lattice. If $H \subseteq A$, the ideal $\langle H\rangle_{\mathbf{A}}^{\mathscr{X}}$ generated by $H$ is the set $\left\{p(\vec{a}, \vec{b}): p(\vec{x}, \vec{y}) \in \mathrm{IT}_{\mathscr{X}}(\vec{y}), \vec{a} \in A \vec{b} \in H\right\}$. In contrast with the previous notation 
we write $(a)_{\mathbf{A}}^{\mathscr{X}}$ whenever $H=\{a\}$. Note that $(0)_{\mathbf{A}}^{\mathscr{X}}=\{0\}$. When $\mathscr{K}$ is $\{\mathbf{A}\}$ (or, equivalently, the variety $\vee \mathbf{A}$ generated by $\mathbf{A})$, then a $\mathscr{X}$-ideal is called an ideal and we drop all the affixes and suffixes in sight.

Using just the definition of ideal term one sees easily that, for any reflexive subalgebra $\mathbf{S}$ of $\mathbf{A} \times \mathbf{A}$,

$$
0 / S=\{a:(0, a) \in S\}
$$

is an ideal of $\mathbf{A}$. Thus $0 / \theta \in \mathrm{I}(\mathbf{A})$ for any $\theta \in \operatorname{Con}(\mathbf{A})$. A set of the form $0 / \theta$ for some $\theta \in \operatorname{Con}(\mathbf{A})$ is called a normal set. Normal sets form an algebraic lattice $\mathrm{N}(\mathbf{A})$ under inclusion. The normal set generated by $X \subseteq A$ is denoted by $N_{\mathrm{A}}(X)$. Note that

$$
N_{\mathrm{A}}(X)=0 / \vartheta_{\mathrm{A}}(X \times\{0\})
$$

and hence $N_{\mathbf{A}}(a)=0 / \vartheta_{\mathbf{A}}(a, 0)$. Of course $\mathrm{N}(\mathbf{A}) \subseteq \mathrm{I}(\mathbf{A}) \subseteq \mathrm{I}_{\mathscr{K}}(\mathbf{A})$ whenever $\mathbf{A} \in \mathscr{K}$ and for any $\mathbf{A} \in \mathscr{K}$ the following are equivalent:

(1) The mapping from $\operatorname{Con}(\mathbf{A})$ into $\mathrm{I}_{\mathscr{X}}$ (A) defined by $\theta \mapsto 0 / \theta$ is a lattice homomorphism.

(2) $\mathrm{N}(\mathrm{A})$ is a sublattice of $\mathrm{I}_{\mathscr{K}}(\mathrm{A})$.

A variety $\mathscr{V}$ (respectively an algebra $A$ ) has normal ideals if $\mathrm{I}_{\mathscr{V}}(\mathbf{A})=\mathrm{N}(\mathbf{A})$ for all $A$ in $\mathscr{V}$ (respectively if $\mathrm{I}(\mathrm{A})=\mathrm{N}(\mathrm{A})$ ). The following are equivalent [1]:

(1) A has normal ideals.

(2) $I=0 / \vartheta(I \times I)$ for $I \in \mathrm{I}(\mathbf{A})$.

(3) $I / \vartheta(J \times J)=I \vee J$ for $I, J \in \mathrm{I}(\mathbf{A})$.

(4) $\vartheta(X \times X)$ is injective from $I(A)$ into $\operatorname{Con}(\mathbf{A})$.

In particular, if $\mathbf{A}$ has normal ideals, then $N_{\mathbf{A}}(a)=(a)_{\mathbf{A}}$.

A class of pointed algebras $\mathscr{K}$ is subtractive if for some binary term $s(x, y)$

$$
s(x, 0) \approx x \quad s(x, x) \approx 0
$$

hold in $\mathscr{K}$. Several characterizations of subtractive varieties can be found in $[1,21,2]$. Here we recall that the following are equivalent:

(1) $\mathscr{V}$ is subtractive.

(2) The congruences of each algebra in $\mathscr{V}$ permute at 0 , that is, for any $\mathbf{A} \in \mathscr{V}$, $\theta, \varphi \in \operatorname{Con}(\mathbf{A})$ and $a \in A$ if $(0, a) \in \theta \circ \varphi$, then $(0, a) \in \varphi \circ \theta$.

(3) For all $\mathbf{A} \in \mathscr{V}$, the mapping $0 /$ is a lattice homomorphism from $\operatorname{Con}(\mathbf{A})$ into I(A).

Moreover any subtractive variety has normal ideals, thus $0 /$ is in fact a lattice epimorphism. A variety $\mathscr{V}$ is ideal-determined [15] if it is subtractive and point regular. In this case $0 /$ is a lattice isomorphism between $\mathrm{I}(\mathbf{A})$ and $\operatorname{Con}(\mathbf{A})$. 
Finally let us emphasize that having normal ideals, being subtractive and being ideal-determined are increasingly stronger conditions. The variety of pointed sets has normal ideals but it is not subtractive. The variety of pseudocomplemented distributive lattices is subtractive but not ideal determined [2].

2.2. Fregean varieties We repeat here the main definition for reader's sake. If $\mathscr{K}$ is a class of pointed algebras, for any $\mathbf{A} \in \mathscr{K}$ we consider the relation $\approx_{\mathrm{A}}$ on $A$ defined by

$$
a \approx_{\mathrm{A}} b \text { if and only if } \vartheta_{\mathrm{A}}(0, a)=\vartheta_{\mathrm{A}}(0, b) .
$$

When $\approx_{\mathrm{A}}=0_{\mathrm{A}}$ for any $\mathbf{A} \in \mathscr{K}$, then $\mathscr{K}$ is congruence orderable in [18]. A congruence orderable 0 -regular variety is Fregean. Note that:

- The variety of Hilbert algebras and the variety of equivalential algebras are Fregean.

- The variety of pointed sets, the variety of lower bounded semilattices and the variety of lower bounded distributive lattices are congruence orderable but non Fregean.

Congruence orderable varieties seem very hard to classify mainly for two reasons.

- The variety of pointed sets is congruence orderable, hence one can hardly expect nice structure theorems for congruence orderable varieties.

- Locally finite congruence orderable varieties exhaust all the spectrum of types in the sense of Hobby-McKenzie's Tame Congruence Theory [17]. The examples above imply that no 'omitting type' theorem is possible for congruence orderable varieties.

The situation changes drastically for Fregean varieties. Congruence regularity for a variety implies in fact congruence modularity and congruence $n$-permutability for some $n$ [16]. On the Tame Congruence Theory side such a variety can have only 2 or 3 in its type set and all tails of minimal sets of algebras therein must be empty. This is a very strong condition whose proof is contained in [17, Theorem 8.7 and Theorem 9.14].

All the examples of Fregean varieties given so far are subtractive. This fact is not entirely coincidental. Both in [20] and in [18] it is stated (explicitly or implicitly) that a lot of the ideal structure of Brouwerian semilattices and equivalential algebras is used to get the representation. This becomes very clear once we express the Fregean property in terms of normal sets. For any algebras $\mathbf{A}$ and $u, v \in A$,

$$
\vartheta_{\mathbf{A}}(u, v)=\vartheta_{\mathbf{A}}(\{u\} \times u / \vartheta(u, v)) .
$$

If $\mathbf{A}$ is pointed, taking $u=0$ and $v=a$ or $v=b$ we get at once

$$
a \approx_{\mathrm{A}} b \text { if and only if } \mathrm{N}_{\mathrm{A}}(a)=\mathrm{N}_{\mathrm{A}}(b) .
$$


Thus, if $\mathbf{A}$ belongs to a subtractive variety and $a, b \in A$

$$
a \approx_{\mathrm{A}} b \text { if and only if }(a)_{\mathrm{A}}=(b)_{\mathrm{A}},
$$

since any subtractive variety has normal ideals. The fact that in a subtractive varieties ideals can be always described via (ideal) terms, propagates nicely to the relation $\approx_{\mathrm{A}}$.

THEOREM 2.1. A congruence orderable subtractive variety $\mathscr{V}$ is Fregean.

PROOF. Let $s(x, y)$ be any term witnessing subtractivity for $\mathscr{V}$. We show that the implication

$$
s(x, y)=s(y, x) \quad \longleftrightarrow \quad x=y
$$

holds in $\mathscr{V}$. Thus $\mathscr{V}$ is 0 -regular and therefore Fregean. The right-to-left implication holds in any subtractive variety, since $s(x, x) \approx 0$. Suppose that $\mathbf{A} \in \mathscr{V}, a, b \in A$ and $s(a, b), s(b, a)=0$. If

$$
u(x, y, z)=s(x, s(s(x, y), z)),
$$

then the equations $u(x, 0,0) \approx 0$ and $u(\bar{x}, y, s(x, y)) \approx x$ both hold in $\mathscr{V}$. In particular, $u(x, y, z)$ is an ideal term in $y, z$ and thus

$$
a=u(a, b, s(a, b))=u(a, b, 0) \in(b)_{\mathrm{A}}, \quad b=u(b, a, s(b, a))=u(b, a, 0) \in(a)_{\mathrm{A}} .
$$

Hence $(a)_{\mathrm{A}}=(b)_{\mathrm{A}}$ and since $\mathscr{V}$ is congruence orderable $a=b$.

The converse of the theorem above is false. There are Fregean varieties that fail to be subtractive. To show that this is the case we need a characterization of congruence orderable varieties that appears in [18]. We reproduce here the short proof.

PROPOSITION 2.2. A pointed variety $\mathscr{V}$ is congruence orderable if and only if for every subdirectly irreducible $\mathbf{A} \in \mathscr{V}$ with monolith $\mu$ we have $|0 / \mu|=2$ and all others $\mu$-blocks are trivial.

PROOF. Let $\mathbf{A}$ be a subdirectly irreducible algebra in a congruence orderable pointed variety $\mathscr{V}$ and let $\mu$ be the monolith of $\mathbf{A}$. If $a, b \in A, a, b \neq 0$ and $(a, b) \in \mu$, then for every $\theta \in \operatorname{Con}(\mathbf{A})$ we have $(0, a) \in \theta$ if and only if $(0, b) \in \theta$. This implies $\vartheta_{\mathrm{A}}(0, a)=\vartheta_{\mathrm{A}}(0, b)$ and, since $\mathscr{V}$ is congruence orderable, we have $a=b$.

Conversely, suppose that $\mathbf{A} \in \mathscr{V}$ and for some $a, b \in A$ we have $\vartheta_{\mathbf{A}}(0, a)=$ $\vartheta_{\mathrm{A}}(0, b)$ but $a \neq b$. Then

$$
0_{\mathrm{A}}<\vartheta_{\mathrm{A}}(a, b) \subseteq \vartheta_{\mathrm{A}}(0, a) \vee \vartheta_{\mathrm{A}}(0, b)=\vartheta_{\mathrm{A}}(0, a)
$$


Pick $\theta$ to be a maximal congruence of $\mathbf{A}$ that does not contain $\vartheta_{\mathbf{A}}(a, b)$. Then $\mathbf{A} / \theta$ is subdirectly irreducible with monolith $\vartheta_{\mathrm{A}}(a, b) \vee \theta / \theta$. Moreover $a / \theta \neq b / \theta$ and they both lie in the same block of the monolith of $\mathbf{A} / \theta$. By hypothesis $0 / \theta \in\{a / \theta, b / \theta\}$ and we may assume $0 / \theta=a / \theta$. But then

$$
\vartheta_{\mathbf{A}}(a, b) \subseteq \vartheta_{\mathbf{A}}(0, a) \subseteq \theta
$$

a contradiction.

The following example of a Fregean variety that is not subtractive is essentially due to Kearnes and Szendrei. We reproduce it here with their kind permission.

EXAMPLE 2.3. Let $\mathbf{B}=\left\langle B,+, \cdot,{ }^{\prime}, 0,1\right\rangle$ be the eight-element Boolean algebra. If we label the three atoms as $a, b$ and $c$, then $B=\left\{0, a, b, c, a^{\prime}, b^{\prime}, c^{\prime}, 1\right\}$. We shall construct an algebra $\mathbf{A}$ that is a polynomial subreduct of $\mathbf{B}$. First $A=B \backslash\left\{a^{\prime}\right\}$; next we define

$$
\begin{aligned}
& d_{1}(x, y)=x y^{\prime} a+x^{\prime} y b, \\
& d_{2}(x, y)=x y^{\prime} a+x^{\prime} y c \\
& m(x, y, z, w)=x y z+x y w+x z w+y z w, \\
& \varepsilon(x, y, z, w)=z\left(x y z^{\prime} w^{\prime}+x y^{\prime} z w^{\prime}+x y^{\prime} z^{\prime} w+x^{\prime} y z w^{\prime}+x^{\prime} y z^{\prime} w+x^{\prime} y^{\prime} z w\right), \\
& t(x, y, z, w)=m(x, y, z, w)+\varepsilon(x, y, z, w) .
\end{aligned}
$$

Let $\mathbf{A}=\left\langle A, d_{1}, d_{2}, t, 0\right\rangle$. It is easy to check that $A$ is closed under $d_{1}, d_{2}$ and $t$ and that $t$ and $m$ are both near-unanimity polynomials (but $A$ is not closed under $m$ ). Let $\mathscr{V}=\mathbb{V}(\mathbf{A})$. Since all operations on $\mathbf{A}$ are Boolean polynomials, the restrictions of the eight congruences of $\mathbf{B}$ to $\mathbf{A}$ are distinct congruences of $\mathbf{A}$. Since $t$ is a near-unanimity term, the variety $\mathscr{V}$ is congruence distributive and so the congruence of $\mathrm{A}$ are exactly the eight congruences mentioned above. It follows that $\mathbf{A}$ is a subdirect product of three two-element algebras and the same can be said of its subalgebras. Hence, via Jonsson's lemma, the only subdirectly irreducible members of $\mathscr{V}$ are the two-element quotients of $\mathbf{A}$. From Proposition 2.2 it follows that $\mathscr{V}$ is congruence orderable.

Let now $\alpha=\vartheta_{\mathrm{A}}(0, a), \beta=\vartheta_{\mathrm{A}}(0, b)$ and $\gamma=\vartheta_{\mathrm{A}}(0, c)$. Then the three subdirectly irreducible algebras in $\mathscr{V}$ are

$$
\mathbf{S}_{1}=\mathbf{A} / \alpha \vee \beta, \quad \mathbf{S}_{2}=\mathbf{A} / \alpha \vee \gamma, \quad \mathbf{S}_{3}=\mathbf{A} / \beta \vee \gamma .
$$

It is easy to check that

$$
\begin{aligned}
& \mathbf{S}_{1} \vDash d_{1}(x, y)=0, \quad d_{2}(x, y)=x^{\prime} y, \\
& \mathbf{S}_{2} \vDash d_{1}(x, y)=x^{\prime} y, \quad d_{2}(x, y)=0, \\
& \mathbf{S}_{3} \vDash d_{1}(x, y)=d_{2}(x, y)=x y^{\prime}
\end{aligned}
$$


and hence $V\left(\mathbf{S}_{i}\right), i=1,2,3$, is congruence distributive, semisimple, congruence orderable and ideal determined. Moreover each $\mathbf{S}_{i}$ satisfies the quasi-identity

$$
d_{1}(x, y)=d_{1}(y, x)=d_{2}(x, y)=d_{2}(y, x)=0 \quad \rightarrow \quad x=y
$$

and hence the quasi-identity holds in $\mathscr{V}$ (since a variety is the quasivariety generated by its subdirectly irreducible algebras). Thus $\mathscr{V}$ is 0 -regular and therefore Fregean. $\mathscr{V}$ is not subtractive, though, since the congruences of $\mathbf{A}$ do not permute at 0 . In fact, the reader can check that in $\mathbf{A},(0,1) \in \alpha \circ(\beta \vee \gamma)$ but $(0,1) \notin(b \vee \gamma) \circ \alpha$. This also shows that the join of three ideal determined subvarieties of a 0 -regular variety does not need to be ideal determined.

Then one is led to wonder if any congruence orderable variety has normal ideals. The answer is again negative and an example is easily found.

EXAMPLE 2.4. Let $\mathscr{S}$ be the variety of lower bounded join semilattices and let us denote the lower bound by 0 . An ideal term $t\left(\vec{x}, y_{1}, \ldots, y_{n}\right)$ in $y_{1}, \ldots, y_{n}$ of $\mathscr{S}$ is just $y_{1} \vee \ldots \vee y_{n}$ and hence any ideal is a subalgebra. Trivial examples show that the variety $\mathscr{S}$ does not have normal ideals. However, if $\mathbf{S} \in \mathscr{S}$ and $a \in S$

$$
N(a)=0 / \vartheta_{\mathrm{S}}(0, \bar{a})=\{b: b \leq a\} .
$$

It follows that if $a \approx_{\mathrm{S}} b$, then $N(a)=N(b)$ and by the above $a=b$, hence $\mathscr{S}$ is congruence orderable.

\section{Equationally definable principal ideals}

A Hilbert algebra [13] is an algebra $\langle A, \rightarrow, 1\rangle$ such that for any $a, b, c \in A$

(1) $a \rightarrow 1=1$;

(2) $a \rightarrow(b \rightarrow a)=1$;

(3) $a \rightarrow(b \rightarrow c) \rightarrow((a \rightarrow b) \rightarrow(a \rightarrow c))=1$;

(4) if $a \rightarrow b=1$ and $b \rightarrow a=1$, then $a=b$.

The following facts are well known:

- The class of Hilbert algebras is a variety $\mathscr{H} \mathscr{I}$ [13].

- Any Hilbert algebra satisfies $1 \rightarrow x \approx x$, hence the variety $\mathscr{H} \mathscr{I}$ is subtractive and, by 4. above, ideal determined.

A Brouwerian semilattice [19] is an algebra $\langle A, \rightarrow, \wedge, 1\rangle$ such that for any $a, b, c \in A$

(1) $\langle A, \wedge, 1\rangle$ is an upper bounded semilattice;

(2) $a \rightarrow a=1$;

(3) $(a \rightarrow b) \wedge a=(b \rightarrow a) \wedge b$; 
(4) $(a \wedge b) \rightarrow c=a \rightarrow(b \rightarrow c)$.

If $\mathbf{A}$ is a Brouwerian semilattice and $a, b, c \in A$, then

$$
c \leq a \rightarrow b \text { if and only if } b \wedge c \leq a
$$

Hence $a \rightarrow b$ is the relative pseudocomplement of $a$ and $b$. The variety $\mathscr{B} \mathscr{S}$ of Brouwerian semilattices is ideal determined. Moreover it can be shown that the class of $\rightarrow$-subreduct of $\mathscr{B} \mathscr{S}$ coincides with the variety $\mathscr{H} \mathscr{I}$ of Hilbert algebras and that the congruences (hence the ideals) of a Brouwerian semilattice coincide with those of its $\rightarrow$-reduct.

A dual Brouwerian semilattice is a join semilattice with dual relative pseudocomplementation, that is, an algebra $\langle A, *, \vee, 0\rangle$ such that

$$
a * b \leq c \quad \text { if and only if } \quad b \leq a \vee c
$$

A dual Hilbert algebra is a *-subreduct of a dual Brouwerian semilattice. The distinction between Brouwerian semilattices (Hilbert algebras) and dual Brouwerian semilattices (dual Hilbert algebras) is purely notational and terminological, like in the more familiar case of meet and join semilattices. However, we deal mainly with (semi)lattices of compact and principal ideals of an algebra. They are pointed ordered structures in which the constant is the smallest element in the ordering, hence we feel that the dual concepts are more suitable in our case.

A pointed class $\mathscr{K}$ of algebras has equationally definable principal ideals (EDPI) if there are terms $p_{i}(x, y), q_{i}(x, y), i=1, \ldots, n$ such that for any $\mathbf{A} \in \mathscr{K}$ and $a, b \in A$

$$
a \in(b)^{\mathrm{A}} \quad \text { if and only if } \quad p_{i}(a, b)=q_{i}(a, b), \quad i=1, \ldots, n
$$

Subtractive varieties with EDPI are investigated at length in [2] and [4]. We collect the main facts about them in the theorem below.

THEOREM 3.1. (1) ([4, Theorem 3.1]) An ideal determined variety has EDPI if and only if it has equationally definable principal congruences.

(2) ([2, Theorem 4.4]) A subtractive variety $\mathcal{V}$ has EDPI if and only if there is a single binary term $p(x, y)$ such that for any $\mathbf{A} \in \mathscr{V}$ and $a, b \in A$

$$
a \in(b)^{\mathbf{A}} \quad \text { if and only if } \quad p(a, b)=0 \text {. }
$$

(3) ([4, Theorem 3.4]) If a subtractive variety has EDPI, then there is a binary term $x * y$ witnessing both subtractivity and EDPI in the above sense.

(4) ([2, Proposition 4.2]) If $\mathscr{V}$ is a subtractive EDPI variety, then for any algebra $\mathbf{A} \in \mathscr{V}$ the join semilattice $\mathrm{CI}(\mathbf{A})$ of compact ideals is a dual Brouwerian semilattice. 
Let $\langle S, \vee, 0\rangle$ be a lower bounded join semilattice. If for some $a, b \in S$ the set $\{c: b \leq a \vee c\}$ has a smallest element, then it is denoted by $a * b$ and called the dual relative pseudocomplement of $a$ and $b$. The following lemma is purely computational and it is a variation on the fact that any dual Hilbert algebra is embeddable into a reduct of a dual Brouwerian semilattice.

LEMMA 3.2. If each two elements from a nonempty subset $H$ of a join semilattice $\mathrm{S}$ have a dual relative pseudocomplement that belongs to $H$, then $\langle H, *\rangle$ is a dual Hilbert algebra.

LEMMA 3.3. Let $\mathscr{V}$ be a subtractive variety with EDPI. There exists a binary term $x * y$ of $\mathscr{V}$ such that the following hold.

(1) For any $\mathbf{A} \in \mathscr{V}, I \in \mathrm{I}(\mathbf{A})$ and $a, b \in A$

$$
a * b \in I \text { if and only if } b \in(a)_{\mathbf{A}} \vee I .
$$

(2) The set $\mathrm{PI}(\mathbf{A})$ of principal ideals of $\mathbf{A}$ is closed under dual relative pseudocomplementation and hence is a dual Hilbert algebra.

(3) The mapping $g:\langle A, *, 0\rangle \rightarrow \mathrm{PI}(\mathbf{A})$ defined by $a \mapsto(a)_{\mathrm{A}}$ is a onto homomorphism (of dual Hilbert algebras) and $\operatorname{ker}(g)=\{(a, b): a * b=b * a=0\}$.

PROOF. By Theorem 3.1(3) there is a binary term $x * y$ witnessing both subtractivity and EDPI for $\mathscr{V}$ and for any $\mathbf{A} \in \mathscr{V}$ the semilattice $\mathrm{CI}(\mathbf{A})$ of compact ideals is a dual Brouwerian semilattice. We denote again by $*$ the dual relative pseudocomplementation in $\mathrm{CI}(\mathrm{A})$. Let $a * b \in I$. Since $\mathscr{V}$ has normal ideals, then $I=0 / \theta$ for some $\theta \in \operatorname{Con}(\mathbf{A})$. Thus, $a / \theta * \beta / \theta=0 / \theta$, which in turn implies that $b / \theta \in(a / \theta)_{\mathbf{A} / \theta}$. So there is an ideal term $t(\vec{x}, y)$ in $y$ such that $b / \theta=t(\vec{u} / \theta, a / \theta)$ for some $\vec{u} \in A$. This in turn implies

$$
b \theta t(\vec{u}, a) \vartheta_{\mathrm{A}}(0, a) t(\vec{u}, 0)=0
$$

and so

$$
b \in 0 /\left(\theta \circ \vartheta_{\mathbf{A}}(0, a)\right)=0 / \vartheta_{\mathbf{A}}(0, a) \vee 0 / \theta=(a)_{\mathbf{A}} \vee I,
$$

where we have used the fact that in subtractive varieties congruences permute at 0 . The converse implication is proved similarly and hence 1 . holds.

From (\#) one sees at once that in $\mathrm{CI}(\mathbf{A})$,

$$
(a)_{\mathbf{A}} *(b)_{\mathbf{A}}=(a * b)_{\mathbf{A}}
$$

and so by Lemma 3.2, the set PI(A) of the principal ideals of $\mathbf{A}$ is a dual Hilbert algebra and 2 . holds. 
Finally $g$ is clearly a homomorphism from $\langle A, *, 0\rangle$ to $\operatorname{PI}(\mathbf{A})$ and by (\#) $(a, b) \in$ $\operatorname{ker}(g)$ if and only if $(a)_{\mathbf{A}}=(b)_{\mathbf{A}}$ if and only if $a \in(b)_{\mathbf{A}}$ and $b \in(a)_{\mathbf{A}}$ if and only if $a * b=b * a=0$.

An $n$-ary operation $f$ on a dual Hilbert algebra $\mathbf{A}$ is compatible if for any $i \leq n$ and any $a, b \in A$

$$
(a * b) *\left((b * a) *\left(\tilde{f}_{i}(a) * \tilde{f}_{i}(b)\right)\right)=0
$$

where $\tilde{f_{i}}(x)=f\left(z_{1}, \ldots, z_{i-1}, x, z_{i+1}, \ldots, z_{n}\right)$. One sees easily that a compatible operation is an operation that preserves all dual Hilbert algebra congruences and viceversa. A dual Hilbert algebra with compatible operations is an algebra $\mathbf{A}=$ $\left\langle A, *, 0, f_{\lambda}\right\rangle_{\lambda \in \Lambda}$, where $\langle A, *, 0\rangle$ is a dual Hilbert algebra and any $f_{\lambda}$ is compatible. It is clear that $\operatorname{Con}(\mathbf{A})=\operatorname{Con}(\langle A, *, 0\rangle)$.

THEOREM 3.4. A pointed variety is congruence orderable, subtractive and has equationally definable principal congruences if and only if it is termwise equivalent to a dual Hilbert algebra with compatible operations.

PROOF. It is obvious that any dual Hilbert algebra with compatible operations is congruence orderable, subtractive and has equationally definable principal congruences.

Conversely let $\mathscr{V}$ be subtractive with EDPI and let $x * y$ be the term witnessing subtractivity and EDPI simultaneously. By Lemma 3.3 for any $\mathbf{A} \in \mathscr{V}$ the mapping $g: a \mapsto(a)_{\mathbf{A}}$ is a homomorphism and $\langle A, *, 0\rangle / \operatorname{ker}(g)$ is a dual Hilbert algebra. If $\mathscr{V}$ is also congruence orderable, then it is Fregean, by Theorem 2.1. It follows that $\operatorname{ker}(g)=\approx_{\mathrm{A}}=0_{\mathrm{A}}$, thus $\langle A, *, 0\rangle$ is a dual Hilbert algebra.

To conclude the proof we must show that any basic operation of $\mathbf{A}$ is compatible in the above sense. Let then $f(x)$ a basic operation, which can be taken to be unary without loss of generality. Let $a, b \in A$ and let

$$
(a * b)_{\mathrm{A}} \vee(b * a)_{\mathrm{A}}=0 / \theta
$$

for some $\theta \in \operatorname{Con}(\mathrm{A})$. Then of course $a / \theta * b / \theta=b / \theta * a / \theta=0 / \theta$ and so $(a / \theta)_{\mathbf{A} / \theta}=(b / \theta)_{\mathbf{A} / \theta}$, implying $(a, b) \in \theta$. It follows that $(f(a), f(b)) \in \theta$ and by the properties of $*$,

$$
f(a) * f(b) \in 0 / \theta=(a * b)_{\mathbf{A}} \vee(b * a)_{\mathbf{A}} .
$$

Now (\#) applied twice gives

$$
(a * b) *((b * a) *(f(a) * f(b)))=0
$$

and hence $f$ is compatible. 


\section{Final remarks}

4.1. Subtractivity cannot be removed from the hypotheses of Theorem 3.4. The variety of lower bounded distributive lattices (which is congruence orderable and has equationally definable principal congruences) is a witness of this fact.

4.2. An investigation on a topic closely connected to Theorem 3.4 has been conducted by Czelakowski and Pigozzi in [12]. They take a more 'abstract logical' approach to the subject and deal mostly with quasivarieties, which are the natural counterpart of deductive systems. An Hilbert quasivariety is a quasivariety which is relatively point regular, congruence orderable and has relative equationally definable principal congruences. The main theorem in [12] is a characterization of Hilbert quasivarieties in the same fashion as Theorem 3.4. Under certain conditions, which turn out to be equivalent to subtractivity, their theorem can be specialized to our result.

4.3. The problem of determining the structure of a generic subtractive congruence orderable variety remains open, though Proposition 2.2 could be of some help. In fact, if $\mathscr{V}$ is a subtractive congruence orderable variety, any binary term $s(x, y)$ witnessing subtractivity for $\mathscr{V}$ turns the 0 -block of the monolith of any subdirectly irreducible algebra $\mathbf{A} \in \mathscr{V}$ either into a (dual) Hilbert algebra or into an equivalential algebra. Therefore a good starting point for solving the general problem would be to find a subtractive congruence orderable variety which is neither congruence permutable, nor has equationally definable principal congruences.

4.4. We have explained in the introduction how Fregean varieties become a subject of a separate investigation and thus form a branch of Universal Algebra. We briefly describe here the existing connections. It is now classical to regard a logic (or a deductive system) on a language as a closure operator $\mathbf{C}$ on the formula algebra $\mathbf{F}$ of that language (see for instance [9] and the bibliography therein). Then one usually writes, for $\Gamma \cup\{\psi\} \subseteq F$,

$$
\Gamma \vdash \psi \text { for } \psi \in \mathrm{C}(\Gamma)
$$

To any subtractive variety $\mathscr{V}$ one can associate a logic in the following way. Let $\mathrm{F}$ be the absolutely free algebra on countably many generators in the language of $\mathscr{V}$; then if $\Gamma, \varphi \in F$

$$
\Gamma \vdash \varphi \text { if and only if } \Gamma\left(a_{1}, \ldots, a_{n}\right)=0 \rightarrow \varphi\left(a_{1}, \ldots, a_{n}\right)=0
$$

for any $\mathbf{A} \in \mathscr{V}$ and $a_{1}, \ldots, a_{n} \in A$. Such logic has been called the ideal assertional logic of $\mathscr{V}$ in [3] (but see also [20] for an earlier treatment and [10] or [5] for the 
latest developments). It turns out that a subtractive variety is Fregean if and only if its (ideal) assertional logic is Fregean and strongly and regularly algebraizable.

However, one should be aware of the fact that the are several ways to 'associate' a logic to a (quasi)variety of algebras, or to get a class of algebras that are amenable to a given deductive system (as one realizes perusing the existing literature). It is in fact perfectly possible for a Fregean variety to be 'associated' (in different but sensible ways) to two different deductive systems one of which is Fregean, but the other is not (an example of this can be found in [14]).

4.5. It is also true (thanks to D. Pigozzi for pointing it out to me) that some of the facts we proved in Section 2 hold in the more general case in which the assertional logic of the variety is protoalgebraic and normal sets are used instead of ideals. However, the subtractivity assumption seems to be crucial for proving all the structural results about dual Hilbert algebras.

\section{Acknowledgments}

I wish to thank first Don Pigozzi, who, while visiting Siena in the Spring of 1996, introduced me to Fregean logics. Without the many helpful discussions I had with him, this paper would not have been written. A big 'thank you' also to Pawel Idziak who made the preliminary draft of [18] available to me and warmly encouraged me to go on with my project.

\section{References}

[1] P. Agliano and A. Ursini, 'Ideals and other generalizations of congruence classes', J. Austral. Math. Soc. Ser. A 53 (1992), 103-115.

[2] —_ 'On subtractive varieties II: general properties', Algebra Universalis 36 (1996), 222-259.

[3] _ _ 'On subtractive varieties III: From ideals to congruences', Algebra Universalis 37 (1997), 296-333.

[4] —_, 'On subtractive varieties IV: definability of principal ideals', Algebra Universalis 38 (1997), 355-389.

[5] G. D. Barbour and J. G. Raftery, 'On quasivarieties of logic, regularity conditions and parametrized algebrizations', Preprint, 1997.

[6] W. J. Blok, P. Köhler and D. Pigozzi, 'On the structure of varieties with equationally definable principal congruences II', Algebra Universalis 18 (1984), 334-379.

[7] W. J. Blok and D. Pigozzi, 'On the structure of varieties with equationally definable principal congruences I', Algebra Universalis 15 (1982), 195-227.

[8] — 'The deduction theorem in algebraic logic', unpublished manuscript, 1989.

[9] _ 'Algebraic semantics for universal horn logic without equality', in: Universal algebra and quasi-group theory (eds. A. Romanowska and J. H. D. Smith) (Heldermann, Berlin, 1992) pp. 1-56. 
[10] W. J. Blok and J. G. Raftery, 'Ideals in quasivarieties of algebras', in: Models, algebras and proofs (eds. X. Caceido and C. H. Montenegro), Lecture Notes in Pure and Appl. Math. 203 (Marcel Dekker, New York, 1999) pp. 167-186.

[11] J. R. Büchi and M. T. Owens, 'Skolem rings and their varieties', in: The collected works of J. Richard Büchi (eds. S. MacLane and D. Siefkes) (Springer, New York, 1990) pp. 161-221.

[12] J. Czelakowski and D. Pigozzi, 'Fregean logics with the multiterm deduction theorem and their algebrization', Preprint.

[13] A. Diego, Sur les algèbres de Hilbert, Collection de Logique Mathematique, Series A, no. 21 (Gauthier-Villars, Paris, 1966).

[14] J. M. Font and M. Rius, 'Tetravalent modal algebras and logics', Mathematics preprint series 191 , (Universitat de Barcelona, Barcelona, 1995).

[15] H. P. Gumm and A. Ursini, 'Ideals in universal algebra', Algebra Universalis 19 (1984), 45-54.

[16] J. Hagemann, 'On regular and weakly regular congruences', Preprint 75, (TH, Darmstadt, 1975).

[17] D. Hobby and R. McKenzie, The structure of finite algebras (tame congruence theory), Contemp. Math. (American Mathematical Society, Providence, RI, 1988).

[18] P. M. Idziak, K. Słomczyńska and A. Wroński, 'Equivalential algebras: a study of Fregean varieties', Preprint, 1996.

[19] P. Köhler, 'Brouwerian semilattices', Trans. Amer. Math. Soc. 68 (1981), 103-126.

[20] D. Pigozzi, 'Fregean algebraic logic', in: Algebraic logic (eds. H. Andréka, J. D. Monk and I. Németi), Colloq. Math. Soc. János Bolyai 54 (North-Holland, Amsterdam, 1991) pp. 473-502.

[21] A. Ursini, 'On subtractive varieties I', Algebra Universalis 31 (1994), 204-222.

Dipartimento di Matematica

Via del Capitano 15

53100 , Siena

Italy

e-mail: agliano@unisi.it 